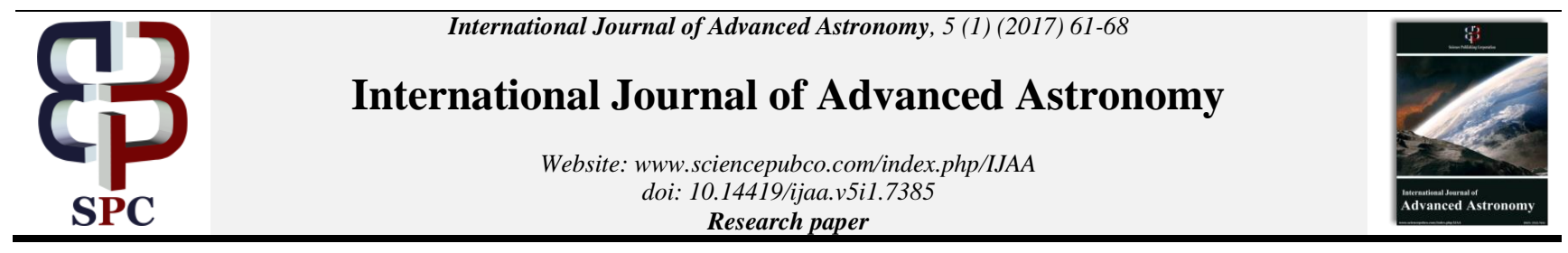

\title{
Modified transformation laws of coordinates and composition of velocities
}

\author{
Mohd. Javed Khilji* \\ 3 D Govindpuri, Thatipur Gwalior 474011, India \\ *Corresponding author E-mail: auqilak@yahoo.co.in
}

\begin{abstract}
A new transformation law of coordinates is determined, which allows the inclusion of complex velocity vectors. It is found that in an isotropic universe which is filled with matter throughout, each frame can have references for its position of rest or of motion in a localized domain wherein a frame at rest does not change its position with time relative to surroundings. However, in the system, the moving frame sees only a reflection of its own motion in the rest-frame to appear it moving in antipodal direction. It is therefore, the relative motion cannot be said to be a just direction reversal with two real velocities having a single magnitude common between them; however, it is a synthesis of both real and imaginary motions. The proposed transformation laws are similar to that of Lorentz under which vector quantities be in cross product or in dot product remain invariant such as Maxwell equations. However, inherent scalar quantities such as mass, time etc. vary to distinguish rest-frame from the moving one. It is seen that the physical processes are exactly the same as Einstein predicted but without reciprocity effects and thus provide a justification of quantization of red-shift and particle decay. These considerations are useful in understanding the intricacies of the micro world such as short-lived particle, antimatter, subatomic particles, gamma rays, gamma-ray burst (GRBs), cosmic rays, dark matter, and asymmetry of Doppler's shifting images.
\end{abstract}

Keywords: Cosmology; Electrodynamics; Lorentz Transformation; Relative Motion; Special Relativity.

\section{Introduction}

Undoubtedly there is no absolute rest or absolute [1] motion in the real world; the rest and motion cannot even be relative terms in an isotropic universe which is filled with baryonic and non-baryonic matter throughout. They are relative terms only if the whole universe is assumed to be made up of mere two frames that do not incorporate any third reference point relative to which either of them can find it at rest [2] or in motion. Obviously in the absence of surroundings none of the frames can ascertain its state of rest if either one is in motion. Each will ascertain its state of rest by seeing the other one in motion. Since the magnitude is common between the two, both the frames are considered moving with the same magnitude but with opposite signs. This phenomenon is associated with a momentary illusion which is common in everyday experience. An observer in stationary train feels his train moving if he sees the other moving train static passing through his train in the close proximity. However, in the same event at any moment if the observer feels his train static, he sees the moving train moving and not static. Thus this phenomenon is associated more with feel-like perceptions than with the true observations. These two diagonally opposite perceptions in the same event signify the smooth transition of states from rest to motion and vice versa for which it gives no justification of any external force that is indispensable for the interchangeability of states according to Newton's first law [2]. Moreover, a single magnitude common between the two frames is shared by the same two frames for their two separate real velocities in the opposite directions in the same event at the same time. This momentary illusion does not last long because the moment the observer refers to surroundings this illusion automatically disappears. Actually the relative motion always shows one type of motion only i.e. the two frames always move opposite to each other. They can never move in the same directions. If the two frames move in the same direction relative to third reference point, on withdrawing the reference point both the frames look moving opposite to each other.

It is often assumed that relative motion with constant velocity finds its practical motivation in reciprocal electrodynamics [3] action of a magnet and a conductor with a phenomenon of deflection in the galvanometer.

The phenomenon is noticeable only when the magnet is partially or fully covered by a coil when either of them is in motion relative to other. The moment they get separated by a distance, though, they are still in relative motion; no phenomenon is noticed. Even in the case of phenomena better results of large deflections are obtained in accelerated mode only, wherein whether a magnet is dropped vertically downward as a free body generating an acceleration ' $\mathrm{g}$ ' into the coil or rotor rotates between the magnets in dynamo or power generators.

At last, to reach to conclusions and to make it generalize, we refer to everyday experiences that are not momentary but a reality and everlasting. Localized domain is the most practical approach to understand the phenomenon of relative motion wherein one frame is at rest relative to surroundings while the other frame moves with respect to it. When seen from the moving frame, the restframe appears moving in antipodal direction. However, when moving frame refers to surroundings it concludes the phenomenon as a mere reflection of its own motion that it sees in the rest-frame for the rest-frame does not change its position relative to surrounding with time. Once it is established that with a single magnitude in the single event, only one frame can move with real velocity (+ve or $-\mathrm{ve}$ ) and in the same event the other one only appears moving cannot have real velocity but imaginary; the results are 
valid to make it generalize. So, mere appearance of motion shows that physical form (i.e. change in position with time) of the motion is nonexistent. For non-real values, we are privileged to have a mathematical tool i.e. $i=\sqrt{-1}$. We therefore, use $i v$ (an imaginary velocity [4] for an appearance of motion, just as the time is conceived as imaginary length in four- dimensional space-time continuum because distance like form of time is non-existent.

\subsection{Non-inherent scalar and inherent scalar quantities}

Non-inherent scalar quantities are vectors, whether they are in cross or in dot product. Inherent scalar quantities are inborn scalar quantities such as mass, time, dimensions, etc., which are directionless. The basic principle of relativity is that one cannot feel any change by oneself without comparing it with the other in the other's state. So any change increase or decrease in vectors will be direction reversal. For example, $\mathrm{B}$ recedes from $\mathrm{A}$ with $+v$ velocity, if B feels motionless at any moment; to him A appears to recede with $-v$. If $+v$ is an increase, $-v$ will be a decrease in vector. So, from both the actions reciprocity is reflected because both feel receding. However, in inherent scalar quantities which are free from directions, any increase or decrease will only be in the magnitude of the quantities. For example, $\mathrm{A}$ at rest observes that $\mathrm{B}$ increases his mass while in motion however if B does not feel any change in him, to him mass of A at rest appears decreasing. Here inherent scalar quantities show de-reciprocity.

\subsection{A Brief discussion of the previous work}

In the previous paper [4] Lorentz, transformation was used wherein the combination of iv and $\mathrm{v}$ was introduced to obtain mass velocity relation in the form of $m(v)=m_{0} \frac{\sqrt{c^{4}-v^{4}}}{c^{2}-v^{2}}$ (showing decre-

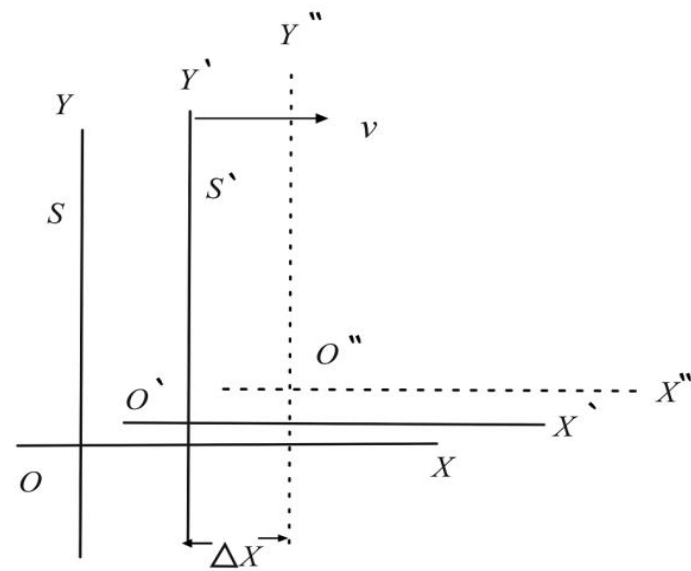

Fig. 1: A

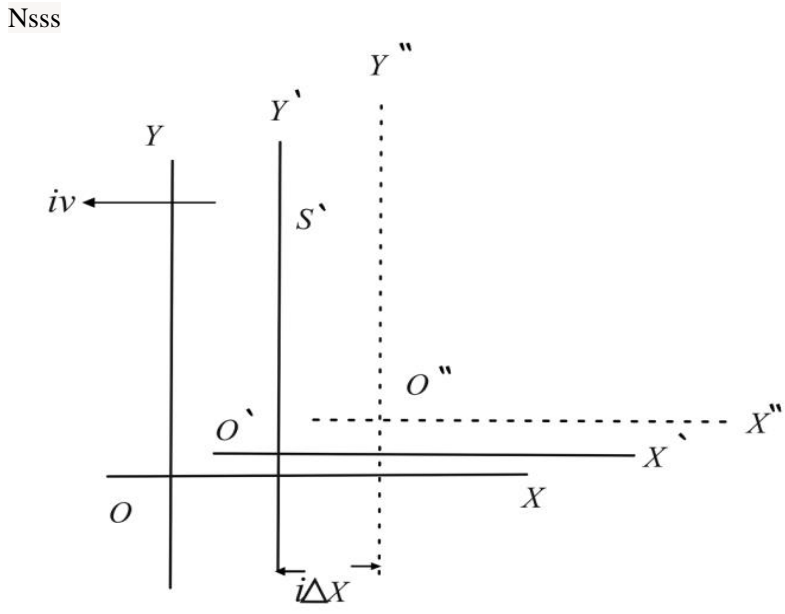

Fig. 1: $B$
Let $S$ and $S^{\prime}$ be two inertial frames of reference (see Fig 1:A \& $1: \mathrm{B}), S^{\prime}$ having uniform velocity $v$ relative to $S$. Let two observers $O$ and $O^{\prime}$ observe any event $\mathrm{P}$ from systems $S$ and $S^{\prime}$ respectively. For convenience let us consider that the $X$-axes of two systems coincide permanently and the velocity is parallel to $X$-axis. The event $\mathrm{P}$ is a light signal and is produced when both $t$ and $t^{\prime}$ are zero and when origins of the two frames coincide. The event $\mathrm{P}$ is determined by co-ordinates $(x, y, z, t)$ and $\left(x^{\prime}, y^{\prime}, z^{\prime}, t^{\prime}\right)$ by observes $O$ and $O^{\prime}$ respectively.

The light pulse produced at $t=0$ will spread out as a growing sphere and the radius of wave front produced in this way will grow with speed $c$, since $(x, y, z, t)$ are co-ordinates of the event from observer in system $S^{\prime}$ at rest, therefore, the equation of spherical surface whose radius grows at the speed $c$, is. ment and increment in mass, i.e. de-reciprocity) and time dilation relation in the form of $\Delta t=\Delta t^{\prime} \frac{\sqrt{c^{4}-v^{4}}}{c^{2}-v^{2}}$ (showing time dilation with time concentration, i.e. de-reciprocity) [4]. The numerator exhibits decrement (relativistic decrease $-R_{d}$ ) while denominator shows increment (relativistic increase $-R_{i}$ ) [5]the paper discussed the kinematic view of relativity. The new relation shows that $R_{i}>R_{d}$ i.e. the moving mass is always greater than its rest mass proper corroborates Einstein's prediction. However, at $c$ $R_{i} \rightarrow \infty$ while $R_{d} \rightarrow 0$ changing the final analysis of infinity to be indeterminate - the mass of photon at $c$. It is corroborated with past experiments where natural pions $\left(\pi^{0}\right)$ when accelerated up to $0.99975 c$, finally they decay into gamma rays. Our theory interprets this decay as pions jumping from $0.99975 c$ to $c$ to transform them into photons. And $\lambda=\frac{h}{m c}$ (de Broglie) shows how shorter [5] and much shorter wavelengths are obtained, giving birth to gamma rays. Similarly, new relation of time dilation with time concentration upholds the asymmetry of Doppler's shifted images, where blue shifted (fast aging) images received by the Ship and red-shifted (slow aging) images are received by Earth; otherwise, both should have been in the same color.

Since, Lorentz transformation has its limitations as it finds its genesis in real velocities alone; modified transformation laws, which accommodates complex velocity vector are derived.

\section{Methodology of modified transformation laws (hypothesis)}

$x^{2}+y^{2}+z^{2}=c^{2} t^{2}$ or $x^{2}+y^{2}+z^{2}-c^{2} t^{2}=0[6]$

Similarly for observer $O^{\prime}$ in system $S^{\prime}$ having the co-ordinates of $\mathrm{P}$ as $\left(x^{\prime}, y^{\prime}, z^{\prime}, t^{\prime}\right)$, the equation of spherical surface is

$x^{12}+y^{12}+z^{12}=c^{2} t^{12}$ or $x^{12}+y^{12}+z^{12}-c^{12} t^{12}=0$

$c$ is considered constant according to special theory of relativity in all frames of references.

As velocity of $S$ is only along $X$-axis: thus forms symmetry and

$y=y^{\prime}$ and $z=z^{\prime}$

Then from eq. (1) and (2) we have

$x^{2}-c^{2} t^{2}=\left(x^{12}-c^{2} t^{12}\right)$ 
Now in frame $S$ the position of $O^{\prime}$ relative to $O$ at any moment $t$ is $x=v t$ or $x-v t=0$ while in frame $S^{\prime}$ the position of $O^{\prime}$ is $x^{\prime}=0$. Here the transformation between $x^{\prime}$ and $x$

Will be $\quad x^{\prime}=k(x-v t)$

$k$, being independent of $x$ and $t$.

Now according to the proposed definition of relative motion, frame $S$ appears to be moving in negative $X$-direction with iv velocity relative to $S^{\prime}$ because frame $S$ is at rest it can only appear to be moving from frame $S^{\prime}$ and that too till the frame $S^{\prime}$ will remain in motion. It is, therefore, the position of observer $O$ relative to $O^{\prime}$ at any time $t$ ' in frame $S^{\prime}$ will be

$x^{\prime}=-i v t^{\prime}$ or $x^{\prime}+i v t^{\prime}=0$

$k$ being independent of $x{ }^{\prime}$ and $t^{\prime}$.

or $x=k\left(x^{\prime}+i v t^{\prime}\right)$

Now substituting the value $x$ ' of from Eq. (5) in Eq. (6) which on solving becomes

$t^{\prime}=k\left[t-\frac{x}{v}\left(1-\frac{1}{k^{2}}\right)\right]$

Now in eq. (4) on putting the value of $x^{\prime}$ from Eq. (5) and value of $t^{\prime}$ from Eq. (7)

We get

$x^{2}-c^{2} t^{2}=k^{2}(x-v t)^{2}-c^{2} k^{2}\left[t-\frac{x}{v}\left(1-\frac{1}{k^{2}}\right)\right]^{2}$

Now on comparing the coefficients of $t^{2}$ of both the sides of equation and similarly on comparing both the sides of coefficients of $2 x t$ we get the same result i.e.

$k=\frac{1}{\sqrt{1-\frac{v^{2}}{c^{2}}}}$

Now putting the value of $k$ in eq. (7)

$$
t^{\prime}=\frac{1}{\sqrt{1-v^{2} / c^{2}}}\left[t-\frac{x}{v} \times \frac{v^{2}}{c^{2}}\right]=\frac{1}{\sqrt{1-v^{2} / c^{2}}}\left(t-\frac{v x}{c^{2}}\right)
$$

And on putting the value of $k$ in the eq. (5)

$$
x^{\prime}=\frac{1}{\sqrt{1-\frac{v^{2}}{c^{2}}}}(x-v t)
$$

Which is the Lorentz transformation with $\mathrm{y}^{\prime}=\mathrm{y}$ and $\mathrm{z}$ '=z.

For inverse transformation,

From eq. (6) putting the value of $x$ in eq. (5) i.e.

$$
x^{\prime}=k\left[k\left(x^{\prime}+i v t^{\prime}\right)-v t\right]
$$

Now on dividing $x^{\prime}=k\left[k\left(x^{\prime}+i v t^{\prime}\right)-v t\right]$ by $i v$ and raising its power to 4 , we get

$$
\left[\frac{k v t}{i v}\right]^{4}=\left[\frac{k^{2} x^{\prime}}{i v}-\frac{x^{\prime}}{i v}+k^{2} t^{\prime}\right]^{4} \quad \text { or }[k t]^{4}=\left[\frac{k^{2} x^{\prime}}{i v}-\frac{x^{\prime}}{i v}+k^{2} t^{\prime}\right]^{4}
$$

Or

$t=k\left[\frac{x^{\prime}}{i v}\left(1-\frac{1}{k^{2}}\right)+t^{\prime}\right]$

Now on putting the value of $x$ from eq. (7) and $t$ from eq. (10) in eq. (4) we get

$$
\begin{gathered}
x^{\prime 2}-c^{2} t^{\prime 2}=k^{2}\left(x^{\prime 2}-2 x^{\prime} i v t^{\prime}+(i v)^{2} t^{\prime 2}\right) \\
-c^{2} k^{2}\left[t^{\prime 2}+\frac{2 x^{\prime} t^{\prime}}{i v}\left(1-\frac{1}{k^{2}}\right)+\frac{x^{2}}{(i v)^{2}}\left(1-\frac{1}{k^{2}}\right)^{2}\right]
\end{gathered}
$$

Now equating the coefficient of $t^{12}$ and $2 x^{\prime} t^{\prime}$ we get the same result i.e.

$$
k=\frac{1}{\sqrt{1-(i v)^{2} / c^{2}}}
$$

Substituting the value of $k$ in eq. (7) we get,

$x=\frac{x^{\prime}+i v t^{\prime}}{\sqrt{1-(i v)^{2} / c^{2}}}$

And putting the value of $k=\frac{1}{\sqrt{1-(i v)^{2} / c^{2}}}$ and $1-\frac{1}{k^{2}}=\frac{-(i v)^{2}}{c^{2}}$ from eq. (12) in eq. (10) we get inverse transformation different from Lorentz's inverse transformation

$t=\frac{t^{\prime}+\frac{i v x^{\prime}}{c^{2}}}{\sqrt{1-(i v)^{2} / c^{2}}}$

\subsection{New composition laws}

However, the proposed paper treats each case differently and provides the formulas accordingly.

Consider two systems $S$ and $S^{\prime}, S^{\prime}$ moving in positive $X$ direction with a velocity $v$ relative to frame $S$. Imagine a particle $P$ moving with $u^{\prime}$ relative to system $S^{\prime}$

Since the Lorentz transformation equations $x{ }^{\prime}=\frac{1}{\sqrt{1-v^{2} / c^{2}}}(x-v t)$;

\&and the transformation equations in this paper are same.

On differentiating eq. (5) and (8)

i.e. equations $x^{\prime}=\frac{1}{\sqrt{1-v^{2} / c^{2}}}(x-v t)$ and $t^{\prime}=\frac{1}{\sqrt{1-v^{2} / c^{2}}}\left(t-\frac{v x}{c^{2}}\right)$

We get

$d x^{\prime}=\frac{1}{\sqrt{1-v^{2} / c^{2}}}(d x-v d t)$

And

$d t^{\prime}=\frac{d t-\frac{v d x}{c^{2}}}{\sqrt{1-v^{2} / c^{2}}}$

Now from Eq. (15) \& (16), on dividing and further the numerator and denominator of their result is divided by $d t$ we get

$u^{\prime}=\frac{u-v}{1-\frac{u v}{c^{2}}}$

Or 


$$
u=\frac{u^{\prime}+v}{1+\frac{u^{\prime} v}{c^{2}}}
$$

1). If a particle moves with $u^{\prime}$ in a reference frame $S^{\prime}$ and if $S^{\prime}$ has a velocity $v$ in negative $\mathrm{X}$-direction relative to $S$ at rest, then the velocity of the particle relative to $S$ will appear to be $-u$.

$-u=\frac{u^{\prime}-v}{1-\frac{u^{\prime} v}{c^{2}}}$

2). If a particle moves with $u^{\prime}$ in a reference frame $S^{\prime}$ and if $S^{\prime}$ has a velocity $v$ in positive X-direction relative to $S$ at rest, then the velocity of the particle relative to $S$ will appear to be $u$

$u=\frac{u^{\prime}+v}{1-\frac{u^{\prime} v}{c^{2}}}$

It is notable that in both the above cases particle is moving in a moving frame $S^{\prime}$ moving with uniform velocity $v$ or $\rightarrow$ relative to $S$ which is at rest. So the particle's velocity what appears to be moving from frame $S$ (at rest) will be the addition of real velocities with their signs, be it negative or positive.

However, the inverse transformation in this paper is different from Lorentz's one as it gives emphasis that the frame at rest is at rest in its own frame and it cannot be considered moving with real velocity, whether, it is negative or positive. It only appears to be moving with the same velocity (with which the moving frame is in motion) from moving frame and that too till it remains in motion so this appearance is attributed to imaginary velocity alone but not to the real one.

Now the inverse transformation according to this paper is;

$$
x=\frac{x^{\prime}+i v t^{\prime}}{\sqrt{1-(i v)^{2} / c^{2}}}
$$

And

$$
t=\frac{x^{\prime}+\frac{i v x^{\prime}}{c^{2}}}{\sqrt{1-(i v)^{2} / c^{2}}}
$$

Now on differentiating the above equations we get

$$
d x=\frac{d x^{\prime}+i v d t^{\prime}}{\sqrt{1-(i v)^{2} / c^{2}}}
$$

$\&$

$$
d t=\frac{d x^{\prime}+\frac{i v d x^{\prime}}{c^{2}}}{\sqrt{1-(i v)^{2} / c^{2}}}
$$

Now from Eq. (21) \& (22), on dividing and further the numerator and denominator of their result is divided by $d t^{\prime}$ we get the relation which is valid for any particle moving with velocity $u^{\prime}$ in a reference frame $S$ at rest relative to $S^{\prime}$ which has a velocity $v$ in negative $\mathrm{X}$ - direction, then the velocity of the particle relative to $S^{\prime}$ will appear to be $u$

$u=\frac{u^{\prime}-i v}{1+\frac{u i v}{c^{2}}}$

Similarly, $u^{\prime}=\frac{u-i v}{1-\frac{u i v}{c^{2}}}$

For a particle moving with velocity $u^{\prime}$ in frame $S$ which is at rest, and its velocity appears to be $u$ in $S^{\prime}$ which is moving with $v$ relative to $S$. It is obvious to note that the motion of a particle in stationary frame will be an addition of velocity [8] and a complex velocity vector with their respective signs.

\section{Maxwell equations are found invariant un- der proposed transformation}

If, $E_{x}, E_{y}, E_{z}$ are components of electric field strength $\mathrm{E}$ along three axes $(x, y, z$,$) respectively relative to S$ and $E_{x}, E_{y}, E_{z}$ relative to $S^{\prime}$ and $B_{x}, B_{y}, B_{z}$ and $B_{x}, B_{y^{\prime}}, B_{z}{ }^{\prime}$ are the components of magnetic field induction B relative to frame $S$ and $S$ ' respectively along the three axes, then

$E_{x^{\prime}}=E_{x}, E_{y^{\prime}}=\frac{E_{y}-(i v) B_{z}}{\sqrt{1+\beta^{2}}} E_{z^{\prime}}=\frac{E_{z}+(i v) B_{y}}{\sqrt{1+\beta^{2}}}$

And

$B_{x^{\prime}}=B_{x}, \quad B_{y^{\prime}}=\frac{B_{y}+(i v) E_{z}}{\sqrt{1+\beta^{2}}} B_{z^{\prime}}=\frac{B_{z}+(i v) E_{y}}{\sqrt{1+\beta^{2}}}$

Maxwell's equations are found invariant under Lorentz transformation [8].

It is to prove that laws of electromagnetic fields are unchanged by the introduction of the proposed law of transformation i.e. Maxwell's equations should be invariant under these transformations.

\subsection{Transformation of differential operators}

Consider two systems $S$ and $S^{\prime}$. $S^{\prime}$ moving with velocity $v$ relative to $S$ along $(+)$ ve direction of $x$-axis.

If a wave is travelling in space with a velocity $v$ in system $S$, then the propagation equation for such a wave is of the form

$\left(\frac{\partial^{2}}{\partial x^{2}}+\frac{\partial^{2}}{\partial y^{2}}+\frac{\partial^{2}}{\partial z^{2}}-\frac{1}{c^{2}} \frac{\partial^{2}}{\partial t^{2}}\right) \psi=0$

Here $\psi$ is known as wave function and differential operator $\left(\frac{\partial^{2}}{\partial x^{2}}+\frac{\partial^{2}}{\partial y^{2}}+\frac{\partial^{2}}{\partial z^{2}}-\frac{1}{c^{2}} \frac{\partial^{2}}{\partial t^{2}}\right)$ Is also known as D'Alembert's operator and is denoted by ${ }^{\circ 2}$ Here $\psi$ is function of $(x, y, z$ and $t)$ thus it may be written as $\psi(x, y, z, t)$

Now in the frame $S^{\prime}$, which is moving relative to $S$, the propagation equation of same wave is given by $\left(\frac{\partial^{2}}{\partial x^{12}}+\frac{\partial^{2}}{\partial y^{12}}+\frac{\partial^{2}}{\partial z^{12}}-\frac{1}{c^{2}} \frac{\partial^{2}}{\partial t^{12}}\right) \psi=0$ where $c$ is not primed because according to principle of relativity, it is always constant.

Thus $\psi$ may be written as $\psi\left(x x^{\prime}, y^{\prime}, z^{\prime}, t^{\prime}\right)$

We, thus have,

$\left(\frac{\partial \psi}{\partial x^{\prime}}=\frac{\partial \psi}{\partial x} \frac{\partial x}{\partial x^{\prime}}+\frac{\partial \psi}{\partial y} \frac{\partial y}{\partial x^{\prime}}+\frac{\partial \psi}{\partial z} \frac{\partial z}{\partial x^{\prime}}+\frac{\partial \psi}{\partial t} \frac{\partial t}{\partial x^{\prime}}\right)$

According to new law of inverse transformations, we have 
$x=\frac{x^{\prime}+i v t^{\prime}}{\sqrt{1-(i v)^{2} / c^{2}}}, Y=Y^{\prime}, Z=Z^{\prime}$ and $t=\frac{x^{\prime}+\frac{i v x^{\prime}}{c^{2}}}{\sqrt{1-(i v)^{2} / c^{2}}}$

From eq. (24) we have

$$
\begin{aligned}
& \frac{\partial x}{\partial x^{\prime}}=\frac{1}{\sqrt{1+\beta^{2}}}, \frac{\partial x}{\partial y^{\prime}}=0, \frac{\partial x}{\partial z^{\prime}}=0 \\
& \frac{\partial x}{\partial t^{\prime}}=\frac{i v}{\sqrt{1+\beta^{2}}} \\
& \frac{\partial x}{\partial x^{\prime}}=\frac{i v / c^{2}}{\sqrt{1+\beta^{2}}}, \frac{\partial y}{\partial y^{\prime}}=1, \frac{\partial z}{\partial z^{\prime}}=0, \frac{\partial t}{\partial y^{\prime}}=0
\end{aligned}
$$

Thus from (23) we have

$$
\begin{aligned}
& \frac{\partial \psi}{\partial x^{\prime}}=\frac{i v / c^{2}}{\sqrt{1+\beta^{2}}}, \frac{\partial \psi}{\partial x}+\frac{i v}{c^{2} \sqrt{1+\beta^{2}}} \frac{\partial \psi}{\partial t} \\
& \frac{\partial \psi}{\partial x^{\prime}}=\frac{1}{\sqrt{1+\beta^{2}}}\left(\frac{\partial}{\partial x}+\frac{i v}{c^{2}} \frac{\partial}{\partial t}\right) \psi
\end{aligned}
$$

Or

$$
\frac{\partial}{\partial x^{\prime}}=\frac{1}{\sqrt{1+\beta^{2}}}\left(\frac{\partial}{\partial x}+\frac{i v}{c^{2}} \frac{\partial}{\partial t}\right)
$$

Also

$\left(\frac{\partial \psi}{\partial y^{\prime}}=\frac{\partial \psi}{\partial x} \frac{\partial x}{\partial y^{\prime}}+\frac{\partial \psi}{\partial y} \frac{\partial y}{\partial y^{\prime}}+\frac{\partial \psi}{\partial z} \frac{\partial z}{\partial y^{\prime}}+\frac{\partial \psi}{\partial t} \frac{\partial t}{\partial y^{\prime}}\right)$

Putting values from (25), this gives

$$
\frac{\partial \psi}{\partial y^{\prime}}=\frac{\partial \psi}{\partial y}
$$

Or

$$
\frac{\partial}{\partial y^{\prime}}=\frac{\partial}{\partial y}
$$

Similarly

Again, we have

$$
\begin{aligned}
& \left(\frac{\partial \psi}{\partial t^{\prime}}=\frac{\partial \psi}{\partial x} \frac{\partial x}{\partial t^{\prime}}+\frac{\partial \psi}{\partial y} \frac{\partial y}{\partial t^{\prime}}+\frac{\partial \psi}{\partial z} \frac{\partial z}{\partial t^{\prime}}+\frac{\partial \psi}{\partial t} \frac{\partial t}{\partial t^{\prime}}\right) \\
& =\frac{\partial \psi}{\partial x} \frac{i v}{\sqrt{1+\beta^{2}}}+\frac{\partial \psi}{\partial t} \frac{1}{\sqrt{1+\beta^{2}}}
\end{aligned}
$$

Since

$$
\left[\frac{\partial y}{\partial t^{\prime}}=\frac{\partial z}{\partial t^{\prime}}=0\right]
$$

Therefore

$$
\frac{\partial \psi}{\partial t^{\prime}}=\frac{1}{\sqrt{1+\beta^{2}}}\left(i v \frac{\partial}{\partial x}+\frac{\partial}{\partial y}\right) \psi
$$

Or

$$
\frac{\partial}{\partial t^{\prime}}=\frac{1}{\sqrt{1+\beta^{2}}}\left(i v \frac{\partial}{\partial x}+\frac{\partial}{\partial y}\right)(32)
$$

From (26),(27),(28) and (29) for transformation equations of differential operators, we have

$$
\begin{aligned}
& \frac{\partial}{\partial x^{\prime}}=\frac{1}{\sqrt{1+\beta^{2}}}\left(\frac{\partial}{\partial x}+\frac{i v}{c^{2}} \frac{\partial}{\partial t}\right) \\
& \frac{\partial}{\partial y^{\prime}}=\frac{\partial}{\partial y}, \frac{\partial}{\partial z^{\prime}}=\frac{\partial}{\partial z}
\end{aligned}
$$

And

$$
\frac{\partial}{\partial t^{\prime}}=\frac{1}{\sqrt{1+\beta^{2}}}\left(i v \frac{\partial}{\partial x}+\frac{\partial}{\partial t}\right)
$$

Similar to inverse transformation, transformations may be evaluated to be

$$
\frac{\partial}{\partial x}=\frac{1}{\sqrt{1-\beta^{2}}}\left(\frac{\partial}{\partial x^{\prime}}-\frac{v}{c^{2}} \frac{\partial}{\partial t^{\prime}}\right)
$$

$\frac{\partial}{\partial y}=\frac{\partial}{\partial y^{\prime}}$

$\frac{\partial}{\partial z}=\frac{\partial}{\partial z}$

And

$\frac{\partial}{\partial t}=\frac{1}{\sqrt{1-\beta^{2}}}\left(\frac{\partial}{\partial t^{\prime}}-v \frac{\partial}{\partial x^{\prime}}\right)$

Equation (30) and (31) involve required transformations of differential operators

\subsection{Invariance of $d{ }^{\prime}$ alembertian operator ${ }^{\circ} 2$}

Consider two systems $S$ and $S$ ', $S^{\prime}$ ' moving with velocity $v$ relative to $S$ along (+)ve direction of x-axis. If $\left(x^{\prime}, y^{\prime}, z^{\prime}, t^{\prime}\right)$ and $(x, y, z, t)$ are coordinates of any event in $S$ and $S^{\prime}$

respectively, then

D' Alembertian in systems $S$ is

${ }^{\circ} 2=\frac{\partial^{2}}{\partial^{2} x^{2}}+\frac{\partial^{2}}{\partial^{2} y^{2}}+\frac{\partial^{2}}{\partial^{2} z^{2}}-\frac{1}{c^{2}} \frac{\partial^{2}}{\partial^{2} t^{2}}$

And D' Alembertian in systems $S$ ' is

${ }^{\prime} 2=\frac{\partial^{2}}{\partial^{2} x^{12}}+\frac{\partial^{2}}{\partial^{2} y^{12}}+\frac{\partial^{2}}{\partial^{2} z^{12}}-\frac{1}{c^{2}} \frac{\partial^{2}}{\partial^{2} t^{12}}$

According to transformations of differential operators we have

$\frac{\partial}{\partial x^{\prime}}=\frac{1}{\sqrt{1+\beta^{2}}}\left(\frac{\partial}{\partial x}+\frac{i v}{c^{2}} \frac{\partial}{\partial t}\right)$

$\frac{\partial}{\partial y^{\prime}}=\frac{\partial}{\partial y}, \frac{\partial}{\partial z^{\prime}}=\frac{\partial}{\partial z}$

And

$\frac{\partial}{\partial t^{\prime}}=\frac{1}{\sqrt{1+\beta^{2}}}\left(i v \frac{\partial}{\partial x}+\frac{\partial}{\partial t}\right)$ 
Which gives as

$\frac{\partial^{2}}{\partial^{2} x^{12}}=\frac{1}{\sqrt{1+\beta^{2}}}\left(\frac{\partial^{2}}{\partial^{2} x^{2}}+\frac{(i v)^{2}}{c^{4}} \frac{\partial^{2}}{\partial y^{2}}+\frac{2 i v}{c^{2}} \frac{\partial^{2}}{\partial x} \frac{}{\partial t}\right)$

$\frac{\partial^{2}}{\partial^{2} y^{12}}=\frac{\partial^{2}}{\partial y^{2}}$

And

$\frac{\partial^{2}}{\partial^{2} z^{12}}=\frac{\partial^{2}}{\partial z^{2}}$

And

$\frac{\partial^{2}}{\partial t^{12}}=\frac{1}{\sqrt{1+\beta^{2}}}\left((i v)^{2} \frac{\partial^{2}}{\partial x^{2}}+\frac{\partial^{2}}{\partial t^{2}}+\frac{2 i v}{c^{2}} \frac{\partial^{2}}{\partial x} \frac{-}{\partial t}\right)$

Therefore,

${ }^{0,2}=\frac{\partial^{2}}{\partial x^{12}}+\frac{\partial^{2}}{\partial y^{12}}+\frac{\partial^{2}}{\partial z^{12}}-\frac{1}{c^{2}} \frac{\partial^{2}}{\partial t^{12}}$

$\frac{\partial^{2}}{\partial^{2} x^{12}}=\frac{1}{\sqrt{1+\beta^{2}}}\left(\frac{\partial^{2}}{\partial x^{2}}+\frac{(i v)^{2}}{c^{4}} \frac{\partial^{2}}{\partial y^{2}}+\frac{2 i v}{c^{2}} \frac{\partial^{2}}{\partial x} \frac{-}{\partial t}\right)+\frac{\partial^{2}}{\partial y^{2}}+\frac{\partial^{2}}{\partial z^{2}}$

$-\frac{1}{c^{2}} \frac{1}{1-\beta^{2}}\left((i v)^{2} \frac{\partial^{2}}{\partial t^{2}}+\frac{\partial^{2}}{\partial x^{2}}+2 v \frac{\partial^{2}}{\partial x \partial t}\right)$

$\Rightarrow \frac{\partial^{2}}{\partial x^{2}}\left\{\frac{1}{\sqrt{1+\beta^{2}}}-\frac{\frac{(i v)^{2}}{c^{2}}}{\sqrt{1+\beta^{2}}}\right\}+\frac{\partial^{2}}{\partial y^{2}}+\frac{\partial^{2}}{\partial z^{2}}-\frac{\partial^{2}}{c^{2} \partial t^{2}}\left\{\frac{1}{\sqrt{1+\beta^{2}}}-\frac{\frac{(i v)^{2}}{c^{2}}}{\sqrt{1+\beta^{2}}}\right\}$

$\Rightarrow \frac{\partial^{2}}{\partial x^{2}}+\frac{\partial^{2}}{\partial y^{2}}+\frac{\partial^{2}}{\partial z^{2}}-\frac{\partial^{2}}{c^{2} \partial t^{2}}$ Since $\left(\frac{v^{2}}{c^{2}}\right)=\beta^{2}$

And

$\frac{\partial^{2}}{\partial x^{2}}+\frac{\partial^{2}}{\partial y^{2}}+\frac{\partial^{2}}{\partial z^{2}}-\frac{\partial^{2}}{c^{2} \partial t^{2}}={ }^{0,2}$

Thus we may say that ${ }^{\circ}{ }^{2}$ is invariant under new Transformations of coordinates.

\subsection{The Invariants of the electromagnetic field}

It is significant to mention that under the proposed transformation system we obtain two invariants of the electromagnetic field[9] which are

i). $c^{2} B^{2}-E^{2}$

ii). $E \cdot B$

Invariance of $c^{2} B^{2}-E^{2}$

According to transformations of electric and magnetic field components

$B_{x^{\prime}}=B_{x} E_{x^{\prime}}=E_{x}$

$B_{y,}=\frac{\left.B_{y}+{ }^{(i v}\right)^{2} / c^{2}}{\sqrt{1+\beta^{2}}} E_{y}=\frac{E_{y}-(i v)^{2} / c^{2}}{\sqrt{1+\beta^{2}}}$
$B_{z^{\prime}}=\frac{B_{z}+(i v)^{2} / c^{2}}{\sqrt{1+\beta^{2}}} E_{z^{\prime}}=\frac{E_{z}-(i v)^{2} / c^{2}}{\sqrt{1+\beta^{2}}}$

Therefore,

$c^{2} B^{2}-E^{2}=c^{2} B_{x^{\prime}}{ }^{2}+c^{2} B_{y \cdot}{ }^{2}+c^{2} B_{z^{\prime}}{ }^{2}-\left(E_{x^{\prime}}{ }^{2}+E_{y^{\prime}}{ }^{2}+E_{z^{\prime}}{ }^{2}\right)$

$=c^{2} B_{x}{ }^{2}+c^{2}\left\{\frac{B_{y}+\frac{i v}{c^{2}} E_{x}}{\sqrt{1+\beta^{2}}}\right\}^{2}+c^{2}\left\{B_{x}-\frac{i v}{c^{2}} E_{y}\right\}^{2}$

$-\left[E_{x}^{2}+\left\{\frac{E_{z}+i v B_{y}}{1+\beta^{2}}\right\}^{2}+\left\{\frac{E_{z}+i v B_{y}}{\sqrt{1+\beta^{2}}}\right\}^{2}\right]$

$=c^{2} B_{x}{ }^{2}+c^{2}\left\{\frac{B_{y}+\frac{i v}{c^{2}} E_{z}{ }^{2}+2 \frac{i v}{c^{2}} B_{y} E_{z}}{\sqrt{1+\beta^{2}}}\right\}^{2}+$

$c^{2}\left\{\frac{B_{x}+\frac{i v}{c^{2}} E_{y}{ }^{2}+2 \frac{i v}{c^{2}} E_{y} B_{z}}{\sqrt{1+\beta^{2}}}\right\}^{2}-$

$\left[E_{x}{ }^{2}+\left\{\frac{E_{x}{ }^{2}+i v B_{z}-2 i v E_{y} B_{z}}{\sqrt{1+\beta^{2}}}\right\}+\left\{\frac{E_{z}+(i v)^{2} B_{y}{ }^{2}+2(i v) E_{x} B_{y}}{\sqrt{1+\beta^{2}}}\right\}\right]$

$\Rightarrow c^{2} B_{x}{ }^{2}+\frac{c^{2} B_{y}{ }^{2}+c^{2} B_{z}{ }^{2}-E_{y}{ }^{2}-E_{z}{ }^{2}}{\left(1+\beta^{2}\right)}\left(1-\frac{(i v)^{2}}{c^{2}}\right)-E_{x}{ }^{2}$

$\Rightarrow c^{2} B_{x}{ }^{2}+c^{2} B_{y}{ }^{2}+c^{2} B_{z}{ }^{2}-\left(E_{x^{\prime}}{ }^{2}+E_{y^{\prime}}{ }^{2}+E_{z^{\prime}}{ }^{2}\right)$

$\Rightarrow c^{2} B^{2}-E^{2}$

ii) $E^{\prime} \cdot B^{\prime}=\left(i E_{x^{\prime}}+j E_{y^{\prime}}+k E_{z^{\prime}}\right) \cdot\left(i B_{x^{\prime}}+j B_{y^{\prime}}+k B_{z^{\prime}}\right)$

$=E_{x}, B_{x},+E_{y} \cdot B_{y},+E_{z} \cdot B_{z}$

$\left\{\frac{\left(E_{x}+\mathrm{iv} B_{v}\right.}{\sqrt{1+\beta}}\right\}\left\{\frac{B_{s}-\left(\mathrm{iv} / c^{2}\right) E_{2}}{\sqrt{1+\beta^{2}}}\right\}$

$E_{x} B_{x}+\left\{\frac{\left(E_{y}-i v B_{x}\right.}{\sqrt{1+\beta^{2}}}\right\}\left\{\frac{B_{y}+\left(i v / c^{2}\right) E_{z}}{\sqrt{1+\beta^{2}}}\right\}+$

$\frac{E_{z} B_{z}-\frac{i v}{c^{2}} E_{y} E_{z}+(i v) B_{z} B_{y}-\frac{i v}{c^{2}} B_{y} E_{y}}{\left(1+\beta^{2}\right)}$

$\Rightarrow E_{x} B_{x}+\frac{E_{y} B_{y}+B_{z} E_{z}}{\left(1+\beta^{2}\right)}\left(1-\frac{(i v)^{2}}{c}\right)$

$\Rightarrow E_{x} B_{x}+E_{y} B_{y}+E_{z} B_{z}=E \cdot B$

\section{Invariance of space-time under modified transformation law}

We will see how the space-time interval is invariant to the proposed transformation laws.

Let the coordinates of two points are $\left(x_{1}, y_{1}, z_{1}, t_{1}\right)$ and $\left(x_{2}, y_{2}, z_{2}, t_{2}\right)$ respectively, the space-time interval is obviously 
$c^{2}\left(t_{2}-t_{1}\right)^{2}-\left(x_{2}-x_{1}\right)^{2}-\left(y_{2}-y_{1}\right)^{2}-\left(z_{2}-z_{1}\right)^{2}$

[Lorentz]. That is also invariant to the proposed transformation. For, if the corresponding coordinates of these points in a frame $S^{\prime}$, moving along the axis of $X$ of frame $S$, with a constant velocity $v$ relative to it, be $\left(x_{1}^{\prime}, y_{1}^{\prime}, z_{1}^{\prime}, t_{1}^{\prime}\right)$ and $\left(x_{2}^{\prime}, y_{2}^{\prime}, z_{2}^{\prime}, t_{2}^{\prime}\right)$ we have, in accordance with inverse transformation under proposed transformation,

$$
\begin{aligned}
& S_{12}^{\prime 2}=c^{2}\left(t_{2}-t_{1}\right)^{2}=c^{2} k^{2}\left[\left(t_{2}^{\prime}-t_{1}^{\prime}\right)+i v\left(x_{2}^{\prime}-x_{1}^{\prime}\right) / c^{2}\right]^{2} \\
& \left(x_{2}-x_{1}\right)^{2}=c k^{2}\left[\left(x_{2}^{\prime}-x_{1}^{\prime}\right)+i v\left(t_{2}^{\prime}-t_{1}^{\prime}\right)\right]^{2},\left(y_{2}-y_{1}\right)^{2}=\left(y_{2}^{\prime}-y_{1}^{\prime}\right)^{2}, \\
& \left(z_{2}-z_{1}\right)^{2}=\left(z_{2}^{\prime}-z_{1}^{\prime}\right)^{2} \\
& c^{2}\left(t_{2}-t_{1}\right)^{2}-\left(x_{2}-x_{1}\right)^{2}-\left(z_{2}-z_{1}\right)^{2}=c^{2} k^{2}\left[\left(t_{2}^{\prime}-t_{1}^{\prime}\right)+i v\left(x_{2}^{\prime}-x_{1}^{\prime}\right) / c^{2}\right]^{2}- \\
& c k^{2}\left[\left(x_{2}^{\prime}-x_{1}^{\prime}\right)+i v\left(t_{2}^{\prime}-t_{1}^{\prime}\right)\right]^{2}-\left(y_{2}^{\prime}-y_{1}^{\prime}\right)^{2}-\left(\mathrm{z}_{2}^{\prime}-\mathrm{z}_{1}^{\prime}\right)^{2} \\
& S_{12}^{\prime 2}=c^{2}\left(t_{2}-t_{1}\right)^{2} k^{2}\left(1+\frac{v^{2}}{c^{2}}\right)-\left(x_{2}^{\prime}-x_{1}^{\prime}\right)^{2} k^{2}\left(1+\frac{v^{2}}{c^{2}}\right)
\end{aligned}
$$

Since

$$
k=\left(\frac{1}{\sqrt{1-\frac{(i v)^{2}}{c^{2}}}}\right)
$$

Or

$$
k^{2}=\left(\frac{1}{1+\frac{v^{2}}{c^{2}}}\right)
$$

We have

$$
\begin{aligned}
& k^{2}\left(1+\frac{v^{2}}{c^{2}}\right)=1 \\
& \therefore S_{12}^{\prime 2}=c^{2}\left(t_{2}^{\prime}-t_{1}^{\prime}\right)^{2}-\left(x_{2}^{\prime}-x_{1}^{\prime}\right)^{2}-\left(\mathrm{y}_{2}^{\prime}-\mathrm{y}_{1}^{\prime}\right)^{2}-\left(\mathrm{z}_{2}^{\prime}-\mathrm{z}_{1}^{\prime}\right)^{2}
\end{aligned}
$$

Thus,

$$
\begin{aligned}
& c^{2}\left(t_{2}-t_{1}\right)^{2}-\left(x_{2}-x_{1}\right)^{2}-\left(y_{2}-y_{1}\right)^{2}-\left(z_{2}-z_{1}\right)^{2} \\
& =c^{2}\left(t_{2}^{\prime}-t_{1}^{\prime}\right)^{2}-\left(x_{2}^{\prime}-x_{1}^{\prime}\right)^{2}-\left(\mathrm{y}_{2}^{\prime}-\mathrm{y}_{1}^{\prime}\right)^{2}-\left(\mathrm{z}_{2}^{\prime}-\mathrm{z}_{1}^{\prime}\right)^{2}
\end{aligned}
$$

Or

$$
S_{12}^{2}=S_{12}^{\prime 2}
$$

Which clearly shows that Space-Time interval is invariant to proposed Transformation in this theory.

\subsection{Transformation for rotating frame of reference}

We obtain the same result through the proposed theory which Minkowaski established using Lorentz transformation; because for real velocity the transformation in the proposed theory and Lorentz transformation are not two different but the same. Minkowaski established from a comparison with the transformation equations that the change from a frame $S$ with axes $(x, y, z, t)$ to a frame $S^{\prime}$ with axes $\left(x^{\prime}, y^{\prime}, z^{\prime}, t^{\prime}\right)$ and moving relative to $S$, along the
$X$ axes with a velocity $v$ in time $t$ is equivalent to rotating the axes $(x, y, z, w=i c t)$ through an $\operatorname{angel}(\theta=v t)$ in the $x w$ plan such that to an observer in $S$ this event of rotation with $\theta$ appears to be

$$
\cos \theta=\frac{1}{\sqrt{1-\frac{v^{2}}{c^{2}}}}
$$

Since $v<c, \cos \theta>1$; hence $\theta$ is imaginary.

However, for inverse transformation, Lorentz uses the same real velocity with opposite signs whereas in the proposed theory complex velocity vector is used with opposite signs.

Let us examine if we get the same results with complex velocity vector of the proposed theory.

According to the proposed theory to an observer in moving, frame $S^{\prime}$ frame $S$ (at rest) will appear moving with velocity - $i v$ in the same time $t$ is equivalent to rotating the axes $\left(x^{\prime}, y^{\prime}, z^{\prime}, w^{\prime}=i c t\right)$ through an angel $=(-i v t)$ or $-i \theta$ in the $x w$ plan. Straightaway $(-i \theta)$ is born an imaginary angle.

The angels being imaginary for both the observers prove that the space-time intervals are invariant, which further justifies the existence of forth coordinate of time as an imaginary length along with the three spatial coordinates in the proposed transformation. With this proof, we can convincingly state that it follows the Poincare transformations in the complex forms.

\section{Conclusion}

This paper emphasizes that a single magnitude attached with one frame cannot impart another frame a real velocity in the same event at the same point of time, what moving frame sees is a mere reflection of its own motion in the other frame to appear it moving in antipodal direction. Thus the relative motion is not a just direcstion reversal with real velocities but a synthesis of both real and imaginary motions. The inclusion of real velocity $(+v$ or $-v)$ gives the same Lorentz transformation but iv (+ve or $-\mathrm{ve}$ ) reverses it for inverse transformation. And using iv imaginary angle automatically takes birth, which justifies the forth imaginary coordinate of time proving the invariance of Space-time intervals. Under these laws, Maxwell equations are found invariant because noninherent scalar quantities involve vector quantities, whether they are in cross product or in dot product; whereas inherent scalar quantities do not include any vector one such as mass, time, dimensions, which show de-reciprocity to distinguish frame at rest with one that moving. The inclusion of iv with $\mathrm{v}$ in mass velocity relation shows de-reciprocity but moving mass remains increased at higher velocities and at $c$ indeterminate mass is obtained i.e. the mass of photon. Thus matter transforms it into gamma rays at speed $c$. Time dilation coupled with time concentration produces desirable results.

\section{Acknowledgement}

This work is dedicated to my late parents Kherullah Khilji and Aquila Khilji.

\section{References}

[1] Rafael F., Einstein's space - time: an introduction to special and general relativity Springer P. 1 (2007).

[2] Eringen A. C., Maugin G.A. Electrodynamics of Continua II, Springer - verlag New York Inc. printed in USA, (1990) pp 720,721

[3] Cumming Karen, Priscilla, Law W, Redish E.F, Understanding Physics, PJ Cooney John Wiley \& Sons Inc. N Delhi, 2004, Induction a charge in Magnetic Field, pp 891. 
[4] Khilji, J. M. intrinsic laws of motion are invariant, Physics Essays, 24, (2011). https://doi.org/10.4006/1.3660803.

[5] Khilji, J. M. Modified Field Theory, Journal of Theoretics, Dec. (2004).

[6] Beiser Arthur, 2005, Concept of modern physics, 6th edition, Tata McGrawhills Co., New Delhi, Lorentz transformation, pp 39 to 41.

[7] Hans C. O Hanian, 1994, Principles of Physics, The theory of Special Relativity relativistic combination rule for velocities, , w.w Norton \& company New York, pp 27.

[8] Jackson John David, 2007, Classical Electrodynamics III, invariance of electric charge covariance John Wiley \& sons (Asia) New Delhi, second print pp 553 to 558.

[9] KraneKenneths, ,Modern Physics, Willy India, reprint 2006, pp 65 , review of electromagnetic waves ExB Lorentz Transformation, pp 35. 